\title{
Two preventive multivessel stenting strategy with zotarolimus eluting stents in ST- elevation myocardial infarction patients: 12-month results of randomized trial
}

\begin{abstract}
Background: Preventive multivessel percutaneous coronary interventions ( $\mathrm{PCI}$ ) (in primary and staged revascularization) with second generation drug eluting stents (DES) in patients with STelevation myocardial infarction (STEMI) is unresolved and controversial issue of contemporary interventional cardiology. Twelve-month results of our randomized trial (NCT01781715) presented.

Methods and findings: One-year outcomes of 136 consecutive patients with STEMI and multivessel coronary artery disease (MVCAD) (SYNTAX Score 18.9 \pm 7.7 points) undergoing primary $\mathrm{PCl}$ with zotarolimus-eluting stents (Resolute Integrity ${ }^{\mathrm{TM}}$ Stent, Medtronic) were evaluated. The patients were randomized into two groups of preventive multivessel stenting: Multivessel primary stenting (MPS primary, $\mathrm{n}=67$ ) (the infarct-related artery (IRA) and non-IRA stenting during one $\mathrm{PCl}$ ) and multivessel stenting in staged revascularisation (MSS, n=69) (the IRA only stenting during the primary $\mathrm{PCl}$ and non-IRA stenting during the same hospital period (10.1 \pm 5.1 days between PCls). Over the 12-month observation there were no differences in adverse cardiovascular events among MPS and MSS group. Fatality outcomes in both groups were not exceeded 3\%. No MI and re-PCI survival was 62 (92.5\%) patients in MPS group and 67 $(97.1 \%)$ in MSS group $p>0.05)$. In general study population $(n=136)$ MACE, re-MI, death and stent thrombosis obtained in $5.1 \%, 5.1 \%, 2.9 \%$ and $4.4 \%$ of patients, respectively.

Conclusions: The use of similar latest generation DES and preventive approach (MPS and MSS with 10.1 \pm 5.1 days between procedures) used in index hospitalization period made it possible to obtain satisfactory results of revascularization compared to most similar randomized trials.
\end{abstract}

Keywords: Preventive revascularization STEMI; Primary $\mathrm{PCl}$; Multivessel coronary artery disease; Zotarolimus-eluting stents

\section{Introduction}

The current guidelines recommend culprit vessel revascularization as a standard treatment option in primary percutaneous coronary intervention (PPCI) [1-6]. Nevertheless, patients with ST-segment elevation myocardial infarction (STEMI) and multivessel coronary artery disease (MVCAD) constitute up to $50 \%$ of all STEMI cases [7,8]. As known, MVCAD is associated with an adverse short and longterm outcome after STEMI [9-11]. The definition and criteria of MVCAD, timing for non-culprit vessel revascularization, and a number of other tactical issues are actively discussed in the recent literature [5,6]. There are three established PCI approaches for treatment of MVCAD and STEMI: 1) PPCI of infarct-related artery (IRA) only (culprit vessel revascularization only, CO) with percutaneous coronary intervention (PCI) of non- infarct-related artery based on findings ischemia (spontaneous or during noninvasive stress-testing); 2) multivessel primary stenting (MPS): IRA is opened with the further stenting of nonIRA during the same PPCI procedure; 3) multivessel staged stenting (MSS): the IRA only is treated during the first PPCI procedure with the subsequent complete
Tarasov RS ${ }^{*}$, Ganyukov VI, Barbarash OL and Barbarash LS

State Research Institute for Complex Issue of Cardiovascular Diseases, Kemerovo, Russia Author for correspondence: roman.tarasov@mail.ru Tel: 0079235260446

Submitted: 04 February 2017 Accepted: 06 March 2017 Published online: 11 March 2017 
revascularization during the second intervention.

Earlier results of trials comparing MPS and CO approaches were controversial [12-19], probably due to the heterogeneity of patient samples, variable endpoints, distinct inclusion criteria and different study protocols. European and American Cardiology Societies for 2010-2013 [1-3] recommended limiting PPCI to the vessel with a culprit stenosis with the exception of cardiogenic shock and persistent ischaemia after PCI. Moreover, performance of PPCI in a non-infarct artery was considered as harmful [2].

However, randomized controlled trial results [20-23] demonstrated usefulness and safety of multivessel stenting in patients with STEMI and MVCAD, both with MPS and MSS approaches. The current guidelines were updated by this data [4-6]. MPS approach was tested in two randomized controlled trials: PRAMI (Preventive Angioplasty in Acute Myocardial Infarction) [20] and CvLPRIT (Complete Versus Culprit-Lesion Only Primary PCI) [21]. In PRAMI trial authors concluded that MPS approach significantly reduces the risk of adverse cardiovascular events, as compared to PCI limited to IRA [20]. In the CvLPRIT trial, authors showed that complete revascularization is beneficial for patients with STEMI and MVCAD in comparison with CO approach [21].

MSS approach was also tested in two randomized controlled trials: DANAMI 3 PRIMULTI (Third Danish Study of Optimal Acute Treatment of Patients with ST-segment Elevation Myocardial Infarction) [22] and PRAGUE-13 (Primary Angioplasty in Patients Transferred from General Community Hospital to Specialized PTCA Units With or Without Emergency Thrombolysis) [23]. In the DANAMI 3 PRIMULTI trial, MSS approach was based on the fractional flow reserve value $\leq 0.80$. MSS approach in patients with STEMI and MVCAD reduced the risk of adverse cardiovascular outcomes [22]. However, PRAGUE-13 trial did not find significant differences between MSS and $\mathrm{CO}$ approaches [23].

All these findings provided the possibility for endorsement (class IIb) of MPS and MSS strategies to patients with STEMI and MVCAD by European and American Cardiology Societies since 2014 [4] and 2015 [5], respectively.

However, there is a number of unresolved issues such as stent choice and timing of staged PCI. We justify the use the new generation of zotarolimus-eluting stents (ZES) with choosing MPS or MSS within one hospital period according to our 12-month results of randomized trial.

\section{Methods}

The randomized trial (NCT01781715) was open label, safety and efficacy evaluated. The purpose is to study results and outcomes of 136 STEMI MVCAD patients after MPS or MSS with ZES (Resolute Integrity ${ }^{\mathrm{TM}}$ Stent, Medtronic). The primary endpoints were the following: (1) Death (cardiac and non-cardiac), (2) MI, (3) Revascularization of target vessel (TVR). Secondary endpoints: (1) The combined endpoint of death, MI and TVR, (2) Stent thrombosis (ST).

Between 2011 and 2014 we randomized 136 patients. Under MVCAD was understood diameter stenosis $(\geq 70 \%)$ of two or more arteries and/or significant branches of coronary arteries (diameter $\geq 2.5 \mathrm{~mm}$ ). Inclusion criteria were the following: (1) Age $\geq 18$ years old; (2) Written informed consent prior to revascularization; (3) Multivessel native coronary arteries significant stenosis and primary PCI $\leq 12 \mathrm{~h}$ from STEMI manifestations and ST-segment elevation $\geq 1 \mathrm{~mm}$ in $\geq 2$ limb electrocardiographic leads or precordial leads $\geq 2 \mathrm{~mm}$ ST-segment elevation; (4) Diameter of coronary artery $\geq 2.5 \mathrm{~mm}$; (5) $\geq 30$ minutes chest pain.

Exclusion criteria were as follows: (1) No MVCAD; (2) Cardiogenic shock; (3) Significant left main stenosis ( $\geq 50 \%)$; (4) Contraindication to use heparin, aspirin, clopidogrel, ticagrelor, zotarolimus. Criteria of procedure success were the following: (1) Angiographic residual stenosis $\leq 20 \%$; (2) TIMI flow grade 3 at the end of procedure. All patients had loading doses of dual antiplatelet therapy and unfractioned heparin before PCI started. After PCI patients prescribed dual antiplatelet therapy for 12 months, statins. After coronary angiography patients randomized to study groups: MPS in one procedure or MSS during one hospital period (10.1 \pm 5.1 days). Human research committee of the Institution was approved the protocol of this randomized trial.

We collected data of coronary angiography and PCIs procedures in DICOM CDs. Evaluating the repeat revascularisation we included PCI or coronary artery bypass grafting with the reason for their implementation such as recurrent symptoms, re-MI or significant ischaemia on stress-testing. In MSS group re-revascularisation was only unplanned PCI or coronary artery bypass grafting. For follow-up patients visited our Centre or underwent phone interviews.

ST estimated using appropriate clinical and angiographic criteria (Academic Research Consortium classification) [24]. Clinical criteria were as follows: (1) Acute chest pain $\geq 15 \mathrm{~min}$; (2) ST-segment deviation of $\geq 1 \mathrm{~mm}$ in two contiguous leads. In the cases of ST clinical manifestations patients immediately underwent angiography. ST angiographic criteria were fresh thrombotic occlusion in the area of implanted stent. 
Two preventive multivessel stenting strategy with zotarolimus eluting stents in ST-elevation myocardial infarction patients: 12-Research Article month results of randomized trial

Criteria of re-MI during eighteen hours after index MI was myocardial ischemia with appropriate recurrent ST-segment deviation $\geq 30$ minutes. After eighteen hours re-MI was diagnosed in the case of new Qwaves, new left bundle-branch block, and/or appropriate increasing of the level of troponin and/or MB-creatine kinase fraction.

Variables were presented as mean \pm SD or percentages. MACE evaluated at the date of first adverse cardiovascular event or at the end of follow-up period (12 months). Follow-up was performed in 100\% of study groups' patients. For statistical analysis Chi Squared and Mann Whitney 'U' test in STATISTICA 8.0 (StatSoft, Tulsa, OK, USA) were used.

\section{Results}

The mean age of general population was $59 \pm 10.6$ years (age values ranged from 31 to 88 ). About $70 \%$ of patients were male. Every fifth patient had diabetes mellitus in study cohort (22.1\%). The MPS group included 67 patients, the MSS group 69 patients. The average time between primary PCI and elective procedure, performed in the MSS group in the hospital period, was $10.1 \pm 5.1$ days. We evaluated the results and outcomes in two study groups with preventive strategy of PCI using ZES (MPS primary vs MSS).

Baseline clinical and demographic characteristics in two study groups shows Table 1. Patients of MPS and MSS group had no differences in their characteristics. Male patients with arterial hypertension without any significant manifestations of acute heart failure predominated in both groups of the study sample.

Table 1: Patient clinical and demographic characteristics.

\begin{tabular}{|c|c|c|c|c|c|}
\hline \multirow[t]{2}{*}{ Variables } & \multicolumn{2}{|c|}{ MPS $(n=67)$} & \multicolumn{2}{|c|}{ MSS ( $n=69)$} & \multirow[t]{2}{*}{$\mathbf{P}$} \\
\hline & $\mathrm{n}$ & $\%$ & $\mathrm{n}$ & $\%$ & \\
\hline Age, years & $\begin{array}{l}58.6 \pm 10 . \\
2\end{array}$ & & $\begin{array}{l}59.1 \pm 11 . \\
1\end{array}$ & & 0.6 \\
\hline Male & 48 & 71.6 & 43 & 62.3 & 0.3 \\
\hline LVEF, \% & & $\begin{array}{l}50.7 \pm 9 . \\
2\end{array}$ & & $\begin{array}{l}51.8 \pm 7 \\
3\end{array}$ & 0.5 \\
\hline $\begin{array}{l}\text { Hypertensio } \\
\mathrm{n}\end{array}$ & 64 & 94 & 61 & 88.4 & 0.4 \\
\hline $\begin{array}{l}\text { Diabetes } \\
\text { mellitus }\end{array}$ & 16 & 23.9 & 14 & 20.3 & 0.8 \\
\hline $\begin{array}{l}\text { Peripheral } \\
\text { artery } \\
\text { disease }\end{array}$ & 13 & 19.4 & 20 & 29 & 0.3 \\
\hline Previous MI & 10 & 14.9 & 4 & 5.8 & 0.2 \\
\hline
\end{tabular}

\begin{tabular}{|l|l|l|l|l|l|}
\hline $\begin{array}{l}\text { Previous } \\
\text { stroke }\end{array}$ & 0 & 0 & 2 & 2.9 & 0.5 \\
\hline $\begin{array}{l}\text { Acute heart } \\
\text { failure (Killip } \\
\text { II) }\end{array}$ & 10 & 14.9 & 8 & 11.6 & 0.8 \\
\hline
\end{tabular}

Angiographic and PCI characteristics shows Table 2.

Table 2: Baseline angiographic characteristics and special features of procedures.

\begin{tabular}{|l|l|l|l|l|l|}
\hline Variables & \multicolumn{1}{|l|}{ MPS $(n=67)$} & \multicolumn{3}{|c|}{ MSS (n=69) } & \multicolumn{1}{|c|}{$P$} \\
\hline $\begin{array}{l}\text { 3-vessel } \\
\text { disease }\end{array}$ & 32 & 47.8 & 31 & 44.9 & 0.9 \\
\hline $\begin{array}{l}\text { SYNTAX } \\
\text { Score }\end{array}$ & $19.1 \pm 7.9$ & & $18.6 \pm 7.1$ & & 0.9 \\
\hline $\begin{array}{l}\text { SYNTAX } \\
\text { Score } \geq 23 \\
\text { points }\end{array}$ & 18 & 26.9 & 16 & 23.2 & 0.8 \\
\hline $\begin{array}{l}\text { Contrast } \\
\text { medium, } \\
\text { ml }\end{array}$ & $325.8 \pm 110.2$ & & $373 \pm 154.5$ & & 0.06 \\
\hline $\begin{array}{l}\text { Mean } \\
\text { number of } \\
\text { stents }\end{array}$ & $2.6 \pm 0.5$ & $2.7 \pm 0.6$ & & 0.7 \\
\hline $\begin{array}{l}\text { Total mean } \\
\text { stent } \\
\text { length, } \\
\text { mm }\end{array}$ & $57.5 \pm 13,4$ & $58 \pm 16.2$ & & 0.6 \\
\hline $\begin{array}{l}\text { Mean stent } \\
\text { diameter, } \\
\text { mm }\end{array}$ & $3.3 \pm 0,4$ & $3.3 \pm 0,5$ & 0.3 \\
\hline
\end{tabular}

Average SYNTAX Score in the study groups consistent with moderate gradation of severity of coronary lesions and not exceed nineteen points. Nearly every second patient in both groups had three-vessel disease.

It should be noted a significant length of stented segments in each group (more than $57 \mathrm{~mm}$ ). Study groups were comparable in angiographic characteristics and PCI features.

Over the 12 months observation there was no significant differences in frequency of adverse cardiovascular events among groups. Only one noncardiac death from rectal cancer in MSS group occurred. Outcomes with death in both groups not exceeded 3\% (Table 3). 


\begin{tabular}{|c|c|c|c|c|c|}
\hline \multirow[t]{2}{*}{ Variables } & \multicolumn{2}{|c|}{ MPS $(n=67)$} & \multicolumn{2}{|c|}{ MSS $(n=69)$} & \multirow[t]{2}{*}{$P$} \\
\hline & $\mathrm{n}$ & $\%$ & $\mathrm{n}$ & $\%$ & \\
\hline All death & 2 & 3 & 2 & 2.9 & 0.9 \\
\hline $\begin{array}{l}\text { of them within } 30 \\
\text { days }\end{array}$ & 2 & 100 & 1 & 50 & - \\
\hline Cardiac death & 2 & 3 & 1 & 1.4 & 0.6 \\
\hline $\mathrm{MI}$ & 5 & 7.5 & 2 & 2.9 & 0.6 \\
\hline $\begin{array}{l}\text { of them within } 30 \\
\text { days }\end{array}$ & 1 & 20 & 2 & 100 & - \\
\hline TVR & 2 & 3 & 1 & 1.4 & 0.6 \\
\hline $\begin{array}{l}\text { of them within } 30 \\
\text { days }\end{array}$ & 0 & 0 & 0 & 0 & - \\
\hline Non-TVR & 0 & 0 & 1 & 1.4 & 0.9 \\
\hline $\begin{array}{l}\text { of them within } 30 \\
\text { days }\end{array}$ & 0 & 0 & 1 & 100 & - \\
\hline $\begin{array}{l}\text { Combined } \\
\text { endpoint (cardiac } \\
\text { death+MI+TVR) }\end{array}$ & 4 & 5.9 & 3 & 4.3 & 0.7 \\
\hline $\begin{array}{l}\text { Stent thrombosis } \\
\text { (on the number of } \\
\text { patients) }\end{array}$ & 4 & 5.9 & 2 & 2.9 & 0.7 \\
\hline $\begin{array}{l}\text { of them within } 30 \\
\text { days }\end{array}$ & 1 & 25 & 2 & 100 & - \\
\hline
\end{tabular}

Survival free of MI and re-PCI was 62 (92.5\%) patients in MPS group and 67 (97.1\%) in MSS group $\mathrm{p}>0.05)$.

\section{Discussion}

In the presented randomized study of STEMI MVCAD patients underwent preventive multivessel PCI (MPS and MSS (10.1 \pm 5.1 days)) with ZES, we observed satisfactory results in both groups despite the initial severity of patients, including a high frequency of occurrence of diabetes $(22.1 \%)$ and the average length of the stented segment $57.8 \pm 14.6 \mathrm{~mm}$.

According to previous recommendations, it was necessary to perform PCI with stenting IRA only in STEMI patients who had no cardiogenic shock $[24,25]$. These guidelines were based on the hypothesis that MPS may increase the risk of MACE and not reasonable in financial effects compare with one-vessel PCI. Several trials suggest that one-vessel PCI strategy avoiding the longer procedures and its complications with ischemia in non-infarcted myocardial regions, decrease of contrast medium volume and risk of contrast induced nephropathy $[15,18]$.

There is no randomized data to definitely answer the issues about the specific scientific merits of any of approaches (MPS or MSS) [26]. And there is no evidence base for second generation DES in STEMI patients with MVCAD, but in recent years, with the development of new advanced devices the outcome of multivessel PCI has markedly improved $[17,19]$.

The results of several randomized trials impacted on pre-existing recommendations $[1,4,27]$. With the new guidelines on myocardial revascularization (2014 ESC/ EACTS) we have new approach to the revascularization for STEMI MVCAD patients [4]. Despite the fact that in no cardiogenic shock or persistent ischemia patients the primary PCI should be limited to IRA stenting only, in patients with ischemia primary PCI should be also performed for non-IRA during a week after hospitalization (evidence grade IIa, level B) [4]. Moreover, now there is the option to perform non-IRA PCI at the time of primary procedure (evidence IIb class, level B) [20]. This modification of the recommendations appeared due to the PRAMI randomized trial revealed advantages of the preventive PCI in non-IRA [1]. Nevertheless, we still do not know answer the key questions - what patients really need MPS, and whether to complete myocardial revascularization is necessary during the hospital period for STEMI MVCAD patients, what kind of stents better?

The present randomized trial had the purpose to estimate 12-month follow-up for preventive strategies of PCI - MPS and MSS with limited time between procedures (10.1 \pm 5.1 days) using second generation ZES in STEMI MVCAD patients. It was found that preventive approach (MPS or MSS) with Resolute Integrity stents had comparable low risk of adverse cardiovascular events in one-year follow-up period. There is no doubt when compare with literature data. According to published trials, one-year incidence of adverse cardiovascular events (MACE) in STEMI MVCAD patients of general cohort with bare metal stents and DES ranged from 23.9 to $28 \%$, re-MI from 1.6 to $8.8 \%$, death from 3.3 to $6.3 \%$, ST from 1.8 to $4.3 \%[12,15,18]$. In presented randomized trial oneyear MACE, re-MI, death and ST occurred in $5.1 \%$, $5.1 \%, 2.9 \%$ and $4.4 \%$ of patients, respectively. There were no significant differences in MACE between two preventive strategies of PCI groups (MPS or MSS).

It is known that the inflammatory reaction due to plaque instability with thrombosis in STEMI patients is not limited to the IRA, but can involve all coronary arteries [28]. Results obtained in the present study suggest that the preventive approach with multivessel stenting (MPS or MSS) using ZES is promising and safe. Moreover, it is possible that the preventive strategy of PCI with DES may not only have clinical, but also financial advantages over standard approaches by reducing the incidence of adverse cardiovascular events, hospital readmissions and re-PCI. It is obvious that preventive multivessel PCI (MPS or MSS) could increase patients comfort associated with absent of 
unscheduled hospitalizations and PCI. This hypothesis was also confirmed in the PRAMI trial. PRAMI trial results had demonstrated advantages of preventive PCI for non-IRA with significantly reducing the risk of MACE, as compared with PCI of IRA [20].

However, the data on this issue remains controversial. Several randomized trials had no shown differences between standard and preventive approach [29]. There were non-significant reductions of MACE between groups. Of interest are the results of another study. In the trial 214 patients were randomized to three groups: IRA PCI, multivessel preventive PCI, and staged preventive PCI with 40 days between PCIs [17]. The rate of re-PCI was less frequent in the two preventive strategy groups compared with the IRA PCI group ( $11 \%$ and $33 \%$, respectively). But there was a no significant differences in the rate of death $(5 \%$ and $12 \%$, respectively). This study as well as presented confirms the benefits of a preventive approach.

In PRAMI trial, combined endpoint was cardiac death, re-MI, refractory angina. At 23 months of observations combined endpoint occurred in $9 \%$ of patients of MPS group compared to $22 \%$ of patients CO group (hazard ratio (HR): 0.35) [20]. In the CvLPRIT trial, authors showed that MACE (mortality, re-MI, heart failure, and repeat revascularization) at one-year follow-up occurred in $10 \%$ of MPS group patients compared to $21 \%$ of $\mathrm{CO}$ group patients (HR: 0.45 ) [21].

In the DANAMI 3 PRIMULTI trial, MSS approach was based on the fractional flow reserve (FFR) value $\leq 0.80$. Combined endpoint, defined as recurrent MI, all-cause mortality, and ischemia-driven revascularization at 27-month follow-up occurred in $40(13 \%)$ patients treated with MSS approach and in $68(22 \%)$ patients treated with CO approach (HR: 0.56 ; $95 \%$ CI: 0.38 to 0.83 ) [22]. However, PRAGUE-13 trial did not find significant differences between MSS and CO approaches (frequencies of primary composite endpoint including all-cause mortality, recurrent MI, or stroke at 38-month followup were $13.9 \%$ vs. $16.0 \%$, respectively) [23].

Unlike most previous studies comparing the strategies MPS and MSS, in the presented randomized trial we used similar latest generation DES (Resolute Integrity ${ }^{\mathrm{TM}}$ Stent, Medtronic). In addition, the significant difference between the designs of our study was the implementation of a complete revascularization in the time interval, limited to one hospital period. Thus, the use of latest generation DES and preventive approach (MPS and MSS) used in index hospitalization period made it possible to obtain satisfactory results of revascularization compared to most similar studies. We had no obtain significant differences in the incidence of MACE between two preventive MPS and MSS groups. That indicates the special importance of the use of preventive strategy with DES for complete revascularization in the index hospitalization for MVCAD STEMI patients.

\section{Conclusion}

The results of presented randomized trial demonstrated advantages and comparable outcomes of both preventive strategies of PCI (MPS or MSS limited to one hospital period) using second generation ZES. It is important to have objective angiographic criteria, indicating to sufficient volume of revascularization performed in the hospital period with MPS or MSS and to fined criteria for personalized choosing an optimal strategy of revascularization.

\section{Study Limitations}

Our study has some limitations. There is limited cohort of patients included in our randomized trial $(n=136)$. Design of presented study contained only two new preventive strategies of PCI for MVD STEMI patients and there were now any convention strategies of PCI (IRA only stenting or staged interventions with not limited time period between PCIs. So, we compared two study groups and also all cohort patients' results with literature data.

\section{Funding}

The randomized trial was performed as part of the main activities of the Institute in the federal budget of the Russian Federation and in the funding of the Russian Academy of Sciences.

\section{Competing and Conflicting Interests}

The randomized trial was carried out with the help of Medtronic company, which provided drug-eluting stents (Resolute Integrity ${ }^{\mathrm{TM}}$ ).

\section{Executive Summary}

Background: Preventive multivessel percutaneous coronary interventions ( $\mathrm{PCl}$ ) (in primary and staged revascularization) with second generation drug eluting stents (DES) in patients with ST-elevation myocardial infarction (STEMI) is unresolved and 
controversial issue of contemporary interventional cardiology. Twelve-month results of our randomized trial (NCT01781715) presented.

Methods and findings: One-year outcomes of 136 consecutive patients with STEMI and multivessel coronary artery disease (MVCAD) (SYNTAX Score 18.9 \pm 7.7 points) undergoing primary PCI with zotarolimus-eluting stents (Resolute Integrity ${ }^{\mathrm{TM}}$ Stent, Medtronic) were evaluated. The patients were randomized into two groups of preventive multivessel stenting: Multivessel primary stenting (MPS primary, $n=67$ ) (the infarct-related artery (IRA) and non-IRA stenting during one $P C I$ ) and multivessel stenting in staged revascularisation (MSS, $n=69$ ) (the IRA only stenting during the primary $\mathrm{PCl}$ and non-IRA stenting during the same hospital period (10.1 \pm 5.1 days between $\mathrm{PCls}$ ). Over the 12-month observation there were no differences in adverse cardiovascular events among MPS and MSS group. Fatality outcomes in both groups were not exceeded $3 \%$. No MI and re-PCl survival was $62(92.5 \%)$ patients in MPS group and $67(97.1 \%)$ in MSS group $p>0.05)$. In general study population ( $n=136)$ MACE, re-Ml, death and stent thrombosis obtained in $5.1 \%, 5.1 \%, 2.9 \%$ and $4.4 \%$ of patients, respectively.

Conclusions: The use of similar latest generation DES and preventive approach (MPS and MSS with $10.1 \pm 5.1$ days between procedures) used in index hospitalization period made it possible to obtain satisfactory results of revascularization compared to most similar randomized trials.

\section{References}

1. Wijns W, Kolh P, Danchin N, et al. Guidelines on myocardial revascularization: The Task Force on Myocardial Revascularization of the European Society of Cardiology (ESC) and the European Association for Cardio-Thoracic Surgery (EACTS). Eur. Heart. J. 31(20): 2501-2555 (2010).

2. O'Gara PT, Kushner FG, Ascheim DD, et al. 2013 ACCF/AHA guideline for the management of ST-elevation myocardial infarction: executive summary: a report of the American College of Cardiology Foundation/American Heart Association Task Force on Practice Guidelines. Circulation. 127: 529-555 (2013).

3. Steg PG, James SK, Atar D, et al. ESC Guidelines for the management of acute myocardial infarction in patients presenting with ST-segment elevation. Eur. Heart. J. 33: 2569-2619 (2012).

4. Windecker S, Kolh P, Alfonso F, et al. for the Task Force on Myocardial Revascularization of the European Society of Cardiology (ESC) and the European Association for CardioThoracic Surgery (EACTS). 2014 ESC/EACTS guidelines on myocardial revascularization. Eur. Heart. J. 35: 2541-2619 (2014).

5. Levine GN, O'Gara PT, Bates ER, et al. 2015 ACC/AHA/ SCAI focused update on primary percutaneous coronary intervention for patients with ST-elevation myocardial infarction: an update of the 2011 ACCF/AHA/SCAI Guideline for Percutaneous Coronary Intervention and the 2013 ACCF/AHA Guideline for the Management of STElevation Myocardial Infarction: A report of the American College of Cardiology/American Heart Association Task Force on Clinical Practice Guidelines and the Society for Cardiovascular Angiography and Interventions. J. Am. Coll. Cardiol. 67: 1235-1250 (2016).

6. Patel MR, Calhoon JH, Dehmer GJ, et al. ACC/ AATS/AHA/ASE/ASNC/SCAI/SCCT/STS 2016 Appropriate Use Criteria for Coronary Revascularization in Patients With Acute Coronary Syndromes. J. Am. Coll. Cardiol. 69(5): 570-591 (2017).

7. Cardarelli F, Bellasi A, Ou FS, et al. Combined impact of age and estimated glomerular filtration rate on in-hospital mortality after percutaneous coronary intervention for acute myocardial infarction (from the American College of Cardiology National Cardiovascular Data Registry). Am. J. Cardiol. 103: 766-771 (2009).

8. Park DW, Clare RM, Schulte PJ, et al. Extent, location, and clinical significance of non-infarctrelated coronary artery disease among patients with ST-elevation myocardial infarction. JAMA. 312: 2019-2027 (2014).

9. Parodi G, Mernisha G, Valenti R, et al. Five year outcome after primary coronary intervention foracute ST elevationmyocardial infarction: results from a single centre experience. Heart. 91: 1541-1544 (2005).
10. Muller DW, Topol EJ, Ellis SG, et al. Thrombolysis and Angioplasty in Myocardial Infarction (TAMI) Study Group. Multivessel coronary artery disease: a key predictor of shortterm prognosis after reperfusion therapy for acute myocardial infarction. Am. Heart. J. 121: 1042-1049 (1991).

11. Sorajja P, Gersh BJ, Cox DA, et al. Impact of multivessel disease on reperfusion success and clinical outcomes in patients undergoing primary percutaneous coronary intervention for acute myocardial infarction. Eur. Heart. J. 28: 1709-1716 (2007).

12. Hannan EL, Samadashvili Z, Walford G, et al. Culprit vessel percutaneous coronary intervention versus multivessel and staged percutaneous coronary intervention for ST-segment elevation myocardial infarction patients with multivessel disease. J. Am. Coll. Cardiol. Intv. 3: 22-31 (2010).

13. Toma M, Buller CE, Westerhout CM, et al. Nonculprit coronary artery percutaneous coronary intervention during acute ST-segment elevation myocardial infarction: insights from the APEX-AMI trial. Eur. Heart. J. 31: 1701-1707 (2010).

14. Cavender MA, Milford-Beland S, Roe MT, et al. Prevalence, predictors, and in-hospital outcomes of non-infarct artery intervention during primary percutaneous coronary intervention for ST-segment elevation myocardial infarction (from the National Cardiovascular Data Registry). Am. J. Cardiol. 104: 507-513 (2009).

15. Corpus RA, House JA, Marso SP, et al. Multivessel percutaneous coronary intervention in patients with multivessel disease and acute myocardial infarction. Am. Heart. J. 148: 493-500 (2004).

16. Kornowski R, Mehran R, Dangas G, et al. Prognostic impact of staged versus "one-time" multivessel percutaneous intervention in acute myocardial infarction: analysis from the HORIZONS-AMI (harmonizing outcomes with revascularization and stents in acute myocardial infarction) trial. J. Am. Coll. Cardiol. 58: 704-711 (2011).

17. Politi L, Sgura F, Rossi R, et al. A randomised trial of targetvessel versus multi-vessel revascularization in ST-elevation myocardial infarction: major adverse cardiac events during long-term follow-up. Heart. 96: 662-667 (2010).

18. Roe MT, Cura FA, Joski PS, et al. Initial experience with multivessel percutaneous coronary intervention during mechanical reperfusion for acute myocardial infarction. Am. J. Cardiol. 88: 170-173 (2001).

19. Varani E, Balducelli M, Aquilina $M$, et al. Single or multivessel percutaneous coronary intervention in STelevation myocardial infarction patients. Catheter. Cardiovasc. Interv. 72: 927-933 (2008).

20. Wald DS, Morris JK, Wald NJ, et al. Randomized trial of preventive angioplasty in myocardial infarction. N. Engl. J. Med. 369: 1115-1123 (2013).

21. Gershlick AH, Khan JN, Kelly DJ, et al. Randomized trial of complete versus lesion-only revascularization in patients 
Two preventive multivessel stenting strategy with zotarolimus eluting stents in ST-elevation myocardial infarction patients: 12- Research Article month results of randomized trial

undergoing primary percutaneous coronary intervention for STEMI and multivessel Disease: the CvLPRIT trial. J. Am. Coll. Cardiol. 65: 963-972 (2015).

22. Engstrøm T, Kelbæk H, Helquist S, et al. Complete revascularisation versus treatment of the culprit lesion only in patients with ST-segment elevation myocardial infarction and multivessel disease (DANAMI 3-PRIMULTI): an open-label, randomised controlled trial. Lancet. 386: 665-671 (2015).

23. Hlinomaz O. Multivessel coronary disease diagnosed at the time of primary PCI for STEMI: complete revascularization versus conservative strategy: the PRAGUE 13 trial. Paper presented at: EuroPCR Paris, France. (2015).

24. Cutlip DE, Windecker S, Mehran R, et al. Clinical endpoints in coronary stent trials: a case for standardized definitions. Circulation. 115: 2344-2351 (2007).

25. Gabriel S, Stefan K, James, DA, et al. The Task Force on the management of ST-segment elevation acute myocardial infarction of the European Society of Cardiology (ESC). Eur. Heart. J. 15: 202 (2012).

26. Widimsky P, David R. How to treat patients with STelevation acute myocardial infarction and multi-vessel disease? Eur. Heart. J. 16: 202 (2010).

27. Binder RK, Maier W, Luscher TF. Multi-vessel revascularization in ST-segment elevation myocardial infarction: where do we stand? Eur. Heart. J. 37, 217-220 (2016).

28. Goldstein JA, Demetriou D, Grines CL, et al. Multiple complex coronary plaques in patients with acute myocardial infarction. N. Engl. J. Med. 343: 915-922 (2000).

29. Di Mario C, Mara S, Flavio A, et al. Single vs multivessel treatment during primary angioplasty: results of the multicenter randomised HEpacoat for culprit or multivesse stenting for Acute Myocardial Infarction (HELP AMI) Study. Int. J. Cardiovasc. Intervent. 6: 128-133 (2004). 